This is the peer reviewed version of the following article: [F. Cheng, E. S. Marshall, A. J. Young, P. J. Robinson, J.-S. G. Bouillard, A. M. Adawi, N. A. Vermeulen, O. K. Farha, M. R. Reithofer, J. M. Chin, Chem. Eur. J. 2017, 23, 15578.], which has been published in final form at [https://doi.org/10.1002/chem.201703812]. This article may be used for non-commercial purposes in accordance with Wiley Terms and Conditions for Use of Self-Archived Versions.

\title{
Magnetic Control of MOF Crystal Orientation and Alignment
}

\author{
Fei Cheng, ${ }^{[a]+}$ Ellis S. Marshall, ${ }^{[a]+}$ Adam J. Young, ${ }^{[a]+}$ Peter J. Robinson, ${ }^{[a]}$ Jean-Sebastien G. \\ Bouillard, ${ }^{[a]}$ Ali M. Adawi, ${ }^{[a]}$ Nicolaas A. Vermeulen, ${ }^{[b]}$ Omar K. Farha, ${ }^{[b]}$ Michael R. Reithofer, ${ }^{[a][c]}$
} and Jia Min Chin ${ }^{[\mathrm{a}] *}$

\begin{abstract}
Most MOFs possess anisotropic properties, the full exploitation of which necessitates a general strategy for the controllable orientation of such MOF crystals. Current methods largely rely upon layer-by-layer MOF epitaxy or tuning of MOF crystal growth on appropriate substrates, yielding MOFs with fixed crystal orientations. Here, the dynamic magnetic alignment of different MOF crystals (NH2-MIL-53(Al) and NU-1000) is shown. The MOFs were magnetized by electrostatic adsorption of iron oxide nanoparticles, dispersed in curable polymer resins (Formlabs 1+ clear resin / Sylgard® 184), magnetically oriented and fixed by resin curing. The importance of crystal orientation on MOF functionality was demonstrated whereby magnetically aligned NU-1000/Sylgard(B) 184 composite was excited with linearly polarized $405 \mathrm{~nm}$ light, affording an anisotropic fluorescence response dependent on the polarization angle of the excitation beam relative to NU-1000 crystal orientation.
\end{abstract}

Metal organic frameworks (MOFs) have received significant research attention in recent years, due to their wide-ranging applications arising from their tunability at the molecular and mesoscopic scales. ${ }^{[1]}$ Their long range molecular order, versatile physical properties and ability to orient active guest molecules also render MOFs especially attractive for use in optical or microelectronic devices. ${ }^{[2]}$ For example, Falcaro et al. recently demonstrated the centimeter-scale pore alignment of a series of copper-based MOFs by exploiting the alignment of $\mathrm{Cu}(\mathrm{OH})_{2}$

[a] Fei Cheng+, Ellis S. Marshall+, Adam J. Young+, Peter J. Robinson, Jean-Sebastien G. Bouillard, Ali M. Adawi, Michael R. Reithofer, ${ }^{*}$ Jia Min Chin*

Gray Centre for Advanced Materials, School of Mathematics and Physical Sciences, University of Hull, Cottingham Road, Hull, East Riding of Yorkshire, HU6 7RX, United Kingdom

E-mail: j.chin@hull.ac.uk

[b] Nicolaas A. Vermeulen, Omar K. Farha,

Department of Chemistry, Northwestern University, 2145 Sheridan Road, Evanston, IL 60208, United States of America

[c] Michael R. Reithofer*,

Institute of Inorganic Chemistry, University of Vienna, Waehringer Strasse 42, 1090 Vienna, Austria

E-mail: michael.reithofer@univie.ac.at

Supporting information for this article is given via a link at the end of the document. substrates. ${ }^{[3]}$ The resulting MOF films absorbed and aligned dye molecules within the MOF channels, which then demonstrated anisotropic optical fluorescence responses to linearly polarized UV light excitation.

As the molecular architecture of a MOF determines its functionality, it is noteworthy that the majority of MOFs possess non-cubic, anisotropic molecular lattices, thereby affording MOFs distinct physical and chemical properties along different crystallographic directions. ${ }^{[4]}$ To fully exploit these directional properties for various applications such as optics or microelectronics requires the ability to controllably orient and align MOF crystals. However, MOFs are typically synthesized as nano or microcrystals, which are difficult to physically manipulate. Instead, attempts to control the alignment and orientation of MOF crystals have relied upon careful tailoring of MOF crystal growth on suitable substrates. ${ }^{[5]}$ This requires specific conditions such as lattice compatibility between the MOF and the substrate, ${ }^{[3]}$ timeconsuming methods employing layer-by-layer epitaxial growth to produce thin films, ${ }^{[5(\mathrm{a}),(\mathrm{b})]}$ or dense growth of MOF crystals for evolutionary selection, whereby crystallites with their fastest growth direction oriented normal to the substrate plane outgrow other crystallites. ${ }^{[5(\mathrm{c}),(\mathrm{d})]}$ Crucially, the requirement for a substrate to induce and retain crystal alignment precludes the possibility of dynamically controlling the orientation or alignment of freestanding MOF crystals in a fluid or polymer matrix, which would be desirable for integrable processing.

Although the synthesis of magnetised MOF crystals and MOF composite have been the explored in recent years, ${ }^{[6]}$ such as for MOF crystal positioning, ${ }^{[7]}$ there have been no reports of magnetic control of MOF crystal orientation. However, Erb et al. recently demonstrated the alignment of alumina microparticles by electrostatically adsorbing superparamagnetic nanoparticles onto the alumina and exposing the magnetized particles to a magnetic field. ${ }^{[8]}$

We therefore decided to investigate the magnetic alignment of MOF microcrystals, by focusing on magnetic functionalization of rod or needle-shaped MOF microcrystals. Establishment of such a method would allowing scientists to more efficiently exploit the anisotropic properties of MOFs through crystal alignment. Such 
a)

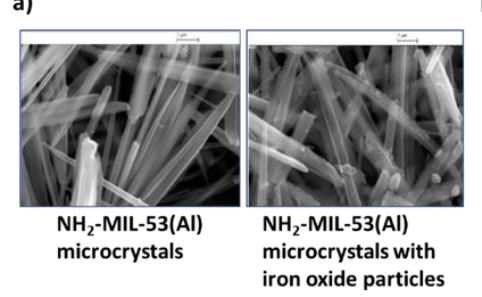

b)
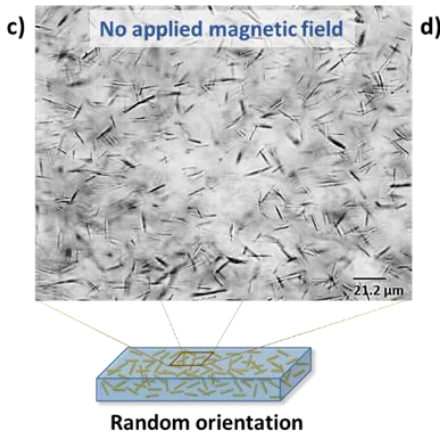

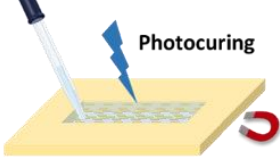

i) Composite casting in silicone mold

ii) Photocuring with/without magnetic alignment

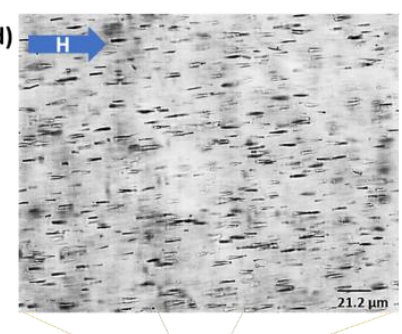

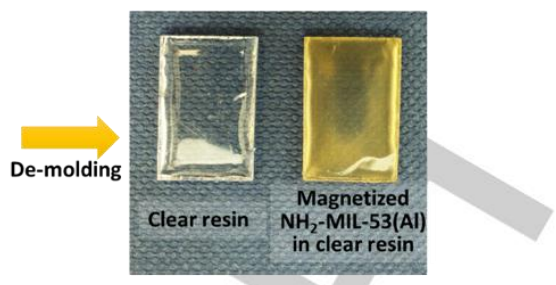
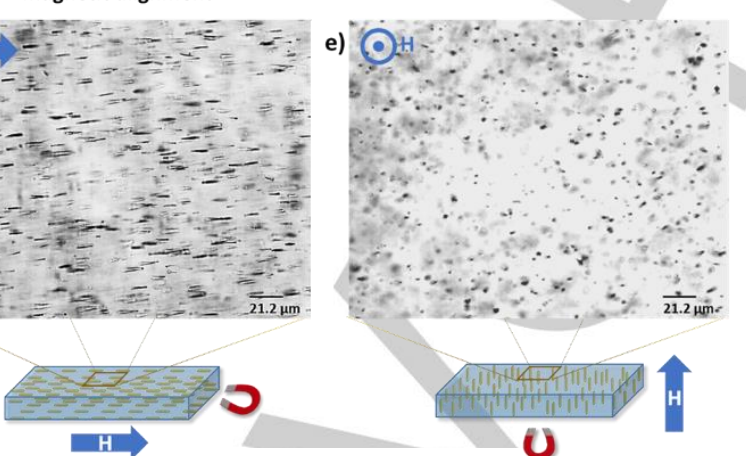

Figure 1. (a) SEM images of $\mathrm{NH}_{2}-\mathrm{MIL}-53(\mathrm{Al})$ microneedles (left) and iron oxide particle-coated $\mathrm{NH}_{2}-\mathrm{MIL}-53(\mathrm{Al})$ microneedles (right). Scale bars represent $1 \mu \mathrm{m}$; (b) illustration of MOF-resin composite preparation and photographs of photocured Form 1+ resin (left) and photocured Form 1+ resin with magnetized $\mathrm{NH}_{2}-\mathrm{MIL}$ 53(Al) microneedles (right). Sample dimensions are $2 \times 6 \times 20 \mathrm{~mm}$; (c) OM images showing $\mathrm{NH}_{2}-\mathrm{MIL}-53$ (Al) microneedles in photocured resin (c-e) with c) random orientation of the MOF particles; d) microneedle alignment along the direction of the applied magnetic field, as indicated by the blue arrow; e) microneedle alignment perpendicular to the major plane of the sample, along the direction of the magnetic field, such that the microneedles appear as dark spots. For (d) and (e), the magnetic field was applied by placing a neodymium magnet $6 \mathrm{~cm}$ from the sample mold.

anisotropically-shaped particles, when magnetized, experience a magnetic torque to align the long-axis of the particles along the direction of an applied magnetic field. ${ }^{[9,8]} \mathrm{NH}_{2}-\mathrm{MIL}-53(\mathrm{Al}) \mathrm{MOF}$ microneedles, which were prepared through coordination modulated growth, ${ }^{[10]}$ were selected as an example of a MOF with crystal shape and lattice anisotropy for this work.

Sodium poly(acrylate)-stabilized iron oxide nanoparticles (3.5 \pm $0.6 \mathrm{~nm}$ in diameter) with an isoelectric point of $\mathrm{pH} 2.8$ (Figure $\mathrm{S} 1$ ) were prepared (refer to experimental section). ${ }^{[11]}$ Above $\mathrm{pH} 2.8$, the nanoparticles bear negative electrostatic charges. $\mathrm{NH}_{2}-\mathrm{MIL}$ 53(Al) crystals, on the other hand, are positively charged below $\mathrm{pH} 10.5$, likely due to protonation of the amine groups on the linker molecules. [ ${ }^{12}$ ] Aqueous suspensions of $\mathrm{NH}_{2}-\mathrm{MIL}-53(\mathrm{Al})$ microneedles ( $8 \pm 2 \mu \mathrm{m}$ in length, aspect ratio of $21 \pm 7$ ) were mixed with the iron oxide nanoparticles at $\mathrm{pH} 3.5$, whereby attractive electrostatic interactions between the two types of particles should prevail to allow nanoparticle attachment to the external MOF particle surfaces. The MOF particles were washed to remove unattached iron oxide nanoparticles and dried for subsequent analysis. Iron oxide particle aggregates on the surface of treated MOF crystals can be observed by SEM (Figure 1a). Further, ICP-MS (Table S1) was utilized to confirm the presence of iron in the treated samples, and this was further supported by TGA analysis (Figure S15). PXRD shows that the $\mathrm{NH}_{2}-\mathrm{MIL}-53(\mathrm{Al})$ microneedles retain their XRD patterns after treatment with iron oxide particles (Figure S8). Magnetized $\mathrm{NH}_{2}-$ MIL-53(Al) treated with iron oxide nanoparticles at $\mathrm{pH} 3.5$ showed mass magnetic susceptibility (Xmass) of $9.09 \times 10^{-6} \mathrm{~m}^{3} \cdot \mathrm{kg}^{-1}$ (Table S1).

Optical microscopy (OM) showed that when aqueous suspensions of the magnetized $\mathrm{NH}_{2}-\mathrm{MIL}-53(\mathrm{Al})$ crystals were placed in a magnetic field, the randomly oriented MOF crystals rapidly align such that their major axes are parallel to the direction of the applied magnetic field, dynamically responding to changes in field direction (see .AVI videos in supporting information). To fix the $\mathrm{NH}_{2}-\mathrm{MIL}-53(\mathrm{Al})$ crystals in their aligned state, the magnetized MOFs were dispersed in a methacrylate-based, photocurable resin (Formlabs 1+ clear resin) using an Ultra-Turrax homogenizer. Magnetic alignment of $\mathrm{NH}_{2}-\mathrm{MIL}-53$ (Al) crystals readily took place even in the viscous resin (dynamic viscosity of $900 \mathrm{cP}$ at $25^{\circ} \mathrm{C}$; that of deionized water is $0.89 \mathrm{cP}$ at $25^{\circ} \mathrm{C}$ ). The resin mixture was degassed, placed into a silicone mold and cured with 395-405 $\mathrm{nm}$ radiation. A neodymium magnet, placed 6 $\mathrm{cm}$ away from the sample, imposed a magnetic field on the sample during curing. OM (Figure $1 \mathrm{c}-e$ ) shows that the MOF crystals are aligned along the applied magnetic field. The orientation distribution of the $\mathrm{NH}_{2}-\mathrm{MIL}-53(\mathrm{Al})$ crystals were plotted (Figure $\mathrm{S} 12$ and $\mathrm{S} 13$ ), where $0^{\circ}$ corresponds to the direction of the magnetic field, if present (refer to ESI for method). Integration of the area under the plots (corresponds to particle percentage distribution) showed that in the absence of a magnetic field, only $16 \%$ of the total area lies within the orientation interval of $0 \pm 10^{\circ}$, whereas significantly improved alignment occurs in the presence of the magnetic field, with $80 \%$ of the total area being in this region. 

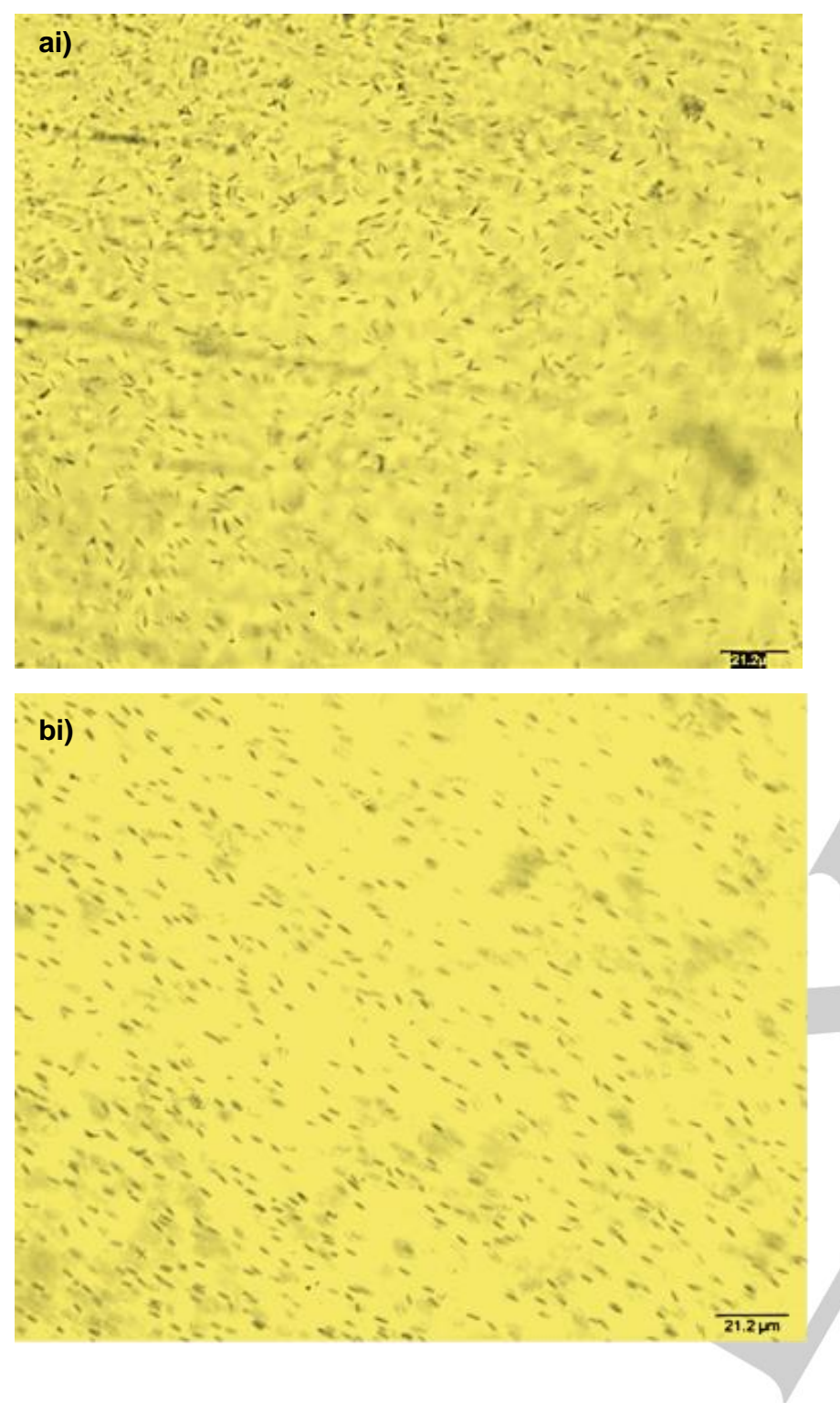
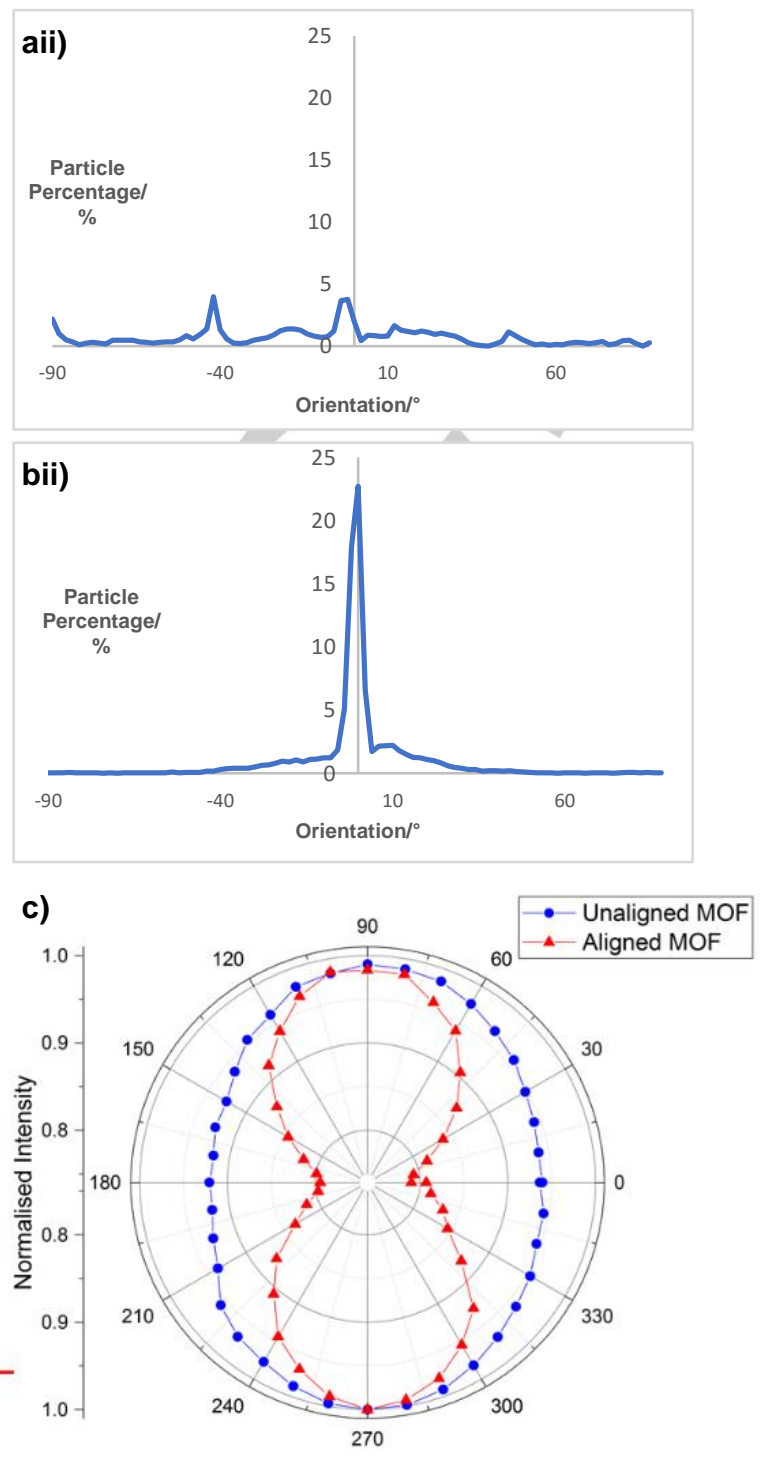

Figure 2. Optical microscope images of (ai) unaligned and (bi) aligned NU-1000 particles in Sylgard ${ }^{\circledR} 184$ and corresponding directionality histograms showing particle percentage vs orientation of (aii) unaligned and (bii) aligned particles; (c) Azimuthal plot of fluorescence intensity response of aligned and unaligned NU1000 particles in Sylgard ${ }^{\circledR} 184$, to linearly polarized light (blue: randomly oriented NU-1000, red: aligned NU-1000).

To investigate the wider applicability of this procedure to other MOFs, we selected NU-1000, a zirconium MOF possessing $31 \AA$ wide mesoporous channels, and high chemical and thermal stability. ${ }^{[13]}$ Microrods of NU-1000 were prepared $(3.7 \pm 0.2 \mu \mathrm{m}$ in length) that bear both lattice and shape anisotropy, with aspect ratios of approximately $5.9 \pm 0.9$. The isoelectric point of NU-1000 occurs at $\mathrm{pH} 4.3,{ }^{[14]}$ whereby the $\mathrm{NU}-1000$ particles in aqueous media are positively charged below this value. Therefore, treating $\mathrm{NU}-1000$ with oppositely charged iron oxide nanoparticles at $\mathrm{pH}$ 3.5 also led to magnetized NU-1000 microrods with $X$ mass $=5.79 x$ $10^{-6} \mathrm{~m}^{3} \cdot \mathrm{kg}^{-1}$. Further, ICP-MS (Table S1) was utilized to confirm the presence of iron in the treated samples, and this was further supported by TGA analysis (Figure S33). The NU-1000 microrods were dispersed in the Formlabs 1+ clear resin, magnetically aligned, and photocured. $\mathrm{OM}$ and plots of NU-1000 particle orientation distribution showed that the presence of a magnetic field led to orientation and alignment of the NU-1000 microrods along the field direction, compared to when the magnetic field was absent (Figure S20 and Figure S21; 69\% of particle distribution within orientation interval $0 \pm 10^{\circ}$ versus $17 \%$, respectively). The $\mathrm{NH}_{2}-\mathrm{MIL}-53(\mathrm{Al})$ and $\mathrm{NU}-1000$ MOFs retained their crystallinity and XRD patterns when embedded in the photocured resin (Figure S8 and S18 respectively). To illustrate the importance of favorable electrostatic interactions between the MOF and iron oxide nanoparticles for successful MOF magnetization, NU-1000 was treated with iron oxide nanoparticles at $\mathrm{pH} 5.9$ whereby electrostatic interactions between NU-1000 and the iron oxide are largely repulsive. As expected, NU-1000 treated under such conditions showed a low $X_{\text {mass }}$ of $0.62 \times 10^{-6} \mathrm{~m}^{3} \mathrm{~kg}^{-1}$.

As mentioned previously, the long-range molecular order arising from the ordered lattices of MOF crystals is useful in optical or electronic applications, whereby molecular orientation is important for efficient energy transfer ${ }^{[3]}$ or charge transport. ${ }^{[15]}$ In NU-1000, the MOF lattice orients the planar pyrene moieties of fluorescent 1,3,6,8-tetrakis( $p$-benzoic acid)pyrene ligands along the $c$-crystallographic axis, which is the major axis of the microrod 
crystals, giving the MOF unique optical properties along this crystallographic axis versus its other axes (Figure S32). ${ }^{[13]}$ It is therefore anticipated that the ability to align NU-1000 crystals within a composite material will afford the overall composite an anisotropic optical response to linearly polarized UV excitation. We therefore investigated the fluorescence response of magnetically aligned NU-1000 microrods to linearly polarized 405 $\mathrm{nm}$ radiation. To avoid fluorescence interference from the polymer matrix, the silicone elastomer Sylgard ${ }^{\circledR} 184$ was used in place of the Formlabs 1+ clear resin (Figure S22-S29). Magnetized NU1000 microrods were dispersed throughout the silicone elastomer by homogenization. The mixture was degassed, placed in a mold, and cured overnight at $40{ }^{\circ} \mathrm{C}$. When the microrods were exposed to a static magnetic field during the curing process, the rods showed a high degree of alignment compared to when a magnetic field was absent (Figure 2). The particle orientation distribution plots show that in the presence of a magnetic field, $73 \%$ of the total area lies within the orientation interval of $0 \pm 10^{\circ}$, whereas only $26 \%$ of the total area lies in this region in the absence of a magnetic field. Fluorescence response studies were carried out whereby the NU-1000/Sylgard ${ }^{\circledR} 184$ samples were irradiated with a $405 \mathrm{~nm}$ linearly polarized UV laser diode at normal incidence to the sample plane. The irradiation polarisation direction was adjusted using a half-wave plate and measurements were obtained at $10^{\circ}$ intervals. The Azimuthal plot (Figure 2c) shows the integrated fluorescence intensity, normalised to its maximum value, as a function of the incident light polarisation orientation. It is observed that the aligned NU-1000/Sylgard ${ }^{\circledR} 184$ sample shows a significant anisotropic response to linearly polarized light, with the highest fluorescence response occurring at $90^{\circ}$, when the incident light polarization is aligned with the major axis of the NU1000 crystals.

In summary, we have demonstrated the magnetic alignment of free-standing MOF crystals, which allows for crystal orientation control in different fluids, such as in a polymeric matrix. The MOF magnetic alignment approach poses several advantages: the MOF crystals i) can be aligned at low volume densities; ii) show short orientation response times; iii) can be dynamically re-

[1] a) P. Falcaro, R. Ricco, A. Yazdi, I. Imaz, S. Furukawa, D. Maspoch, R. Ameloot, J. D. Evans, C. J. Doonan, Coord. Chem. Rev. 2016, 307, 237; b) H. Furukawa, K. E. Cordova, M. O’Keeffe, O. M. Yaghi, Science 2013, 341, 974 and references therein.

[2] I. Stassen, N. Burtch, A. A. Talin, P. Falcaro, M. D. Allendorf, R. Ameloot, Chem. Soc. Rev. 2017, 46, 3185.

[3] P. Falcaro, K. Okada, T. Hara, K. Ikigaki, Y. Tokudome, A. W. Thornton, A. J. Hill, T. Williams, C. Doonan, M. Takahashi, Nat. Mater. 2017, 16, 342.

[4] a) B. V. Harbuzaru, A. Corma, F. Rey, P. Atienzar, J. L. Jordá, H. García, D. Ananias, L. D. Carlos, J. Rocha, Angew. Chem., Int. Ed. 2008, 47, 1080; b) J. C. Tan, A. K. Cheetham, Chem. Soc. Rev. 2011, 40, 1059; c) B. Liu, M. Tu, R. A. Fischer, Angew. Chem. Int. Ed. 2013, 52, 3402.

[5] a) M. C. So, S. Jin, H. J. Son, G. P. Wiederrecht, O. K. Farha, J. T. Hupp, J. Am. Chem. Soc. 2013, 135, 15698; b) O. Shekhah, H. Wang, T. Strunskus, P. Cyganik, D. Zacher, R. Fischer, C. Wöll, Langmuir 2007, 23, 7440; c) T. T. Y. Tan, M. R. Reithofer, E. Y. Chen, A. G. Menon, T. S. A. Hor, J. Xu, J. M. Chin, J. Am. Chem. Soc. 2013, 135, 16272; d) Z. Zhong, J. Yao, R. Chen, Z. Low, M. He, J. Z. Liu, H. J. Wang, J. Mater. Chem. A 2015, 3, 15715.

[6] R. Ricco, L. Malfatti, M. Takahashi, Anita J. Hill, P. Falcaro, J. Mater. Chem. A 2013, 1, 13033. oriented and iv) can be aligned in different fluid matrices. Depending on the fluid matrix utilized, rapid alignment can take place within seconds, thus allowing precise and dynamic control during composite processing. Crucially, this approach also eliminates the requirements for lattice matching of MOF to substrate and the need for careful control of oriented MOF crystal growth. To illustrate the impact of aligning anisotropic MOF crystals on their functional performance, we have shown that a

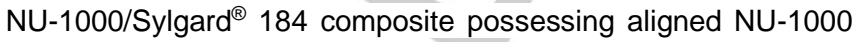
particles gives an anisotropic optical response to linearly polarized excitation light. Although this study relies upon attractive electrostatic interactions between two types of MOFs and iron oxide nanoparticles, the exploitation of such interactions can be easily extended to other MOF/magnetic nanoparticle pairings by adjusting the $\mathrm{pH}$ or other particle environmental factors. The MOF magnetic alignment strategy reported here therefore represents an important addition to scientists' repertoire of tools for controlling MOF crystal orientation which may be of interest for optical applications, or improved separation processes; to this end, we are currently exploring the use of porous gels for water filtration and the fabrication of mixed-matrix membranes for gas purification whereby the MOFs are aligned within the polymer matrix.

\section{Acknowledgements}

J.C. and M.R.R. thank the University of Hull for providing the financial support to carry out this work.

E.S.M., P.J.R. and A.J.Y. thank the University of Hull for the provision of their University Scholarships.

We would also like to thank Dr. Timothy J Prior for helpful discussions on PXRD patterns.

F.C., E.S.M and A.J.Y. contributed equally.

Keywords: Metal-organic framework; fluorescence; anisotropy; magnetic alignment; polymer composites.

[7] a) P. Falcaro, F. Lapierre, B. Marmiroli, M. Styles, Y. Zhu, M. Takahashi, A.J. Hill, C.M. Doherty, J. Mater. Chem. C 2013, 1, 42; b) P. Falcaro, F. Normandin, M. Takahashi, P. Scopece, H. Amenitsch, S. Costacurta, C. M. Doherty, J. S. Laird, M. D. H. Lay, F. Lisi, A. J. Hill, D. Buso, Adv. Mater. 2011 23, 3901.

[8] R. M. Erb, R. Libanori, N. Rothfuchs, A. R. Studart, Science 2012, 335, 199. [9] J. A. Beardslee, B. Sadtler, N. S. Lewis, ACS Nano 2012, 6, 10303.

[10] J. M. Chin, E. Y. Chen, A. G. Menon, H. Y. Tan, T. S. A. Hor, M. K. Schreyer, J. Xu, CrystEngComm 2013, 15, 654.

[11] C.-L. Lin, C.-F. Lee, W.-Y. Chiu, J. Colloid Interf. Sci. 2005, 291, 411. [12] C. Li, Z. Xiong, J. Zhang, C. Wu, J. Chem. Eng. Data 2015, 60, 3414.

[13] J. E. Mondloch, W. Bury, D. Fairen-Jimenez, S. Kwon, E. J. DeMarco, M. H. Weston, A. A. Sarjeant, S. T. Nguyen, P. C. Stair, R. Q. Snurr, O. K. Farha, J. T. Hupp, J. Am. Chem. Soc. 2013, 135, 10294.

[14] P. Li, J. A. Modica, A. J. Howarth, E. L. Vargas, P. Z. Moghadam, R. Q. Snurr, M. Mrksich, J. T. Hupp, O. K. Farha, Chem. 2016, 1, 154.

[15] A. A. Talin, A. Centrone, A.C. Ford, M.E. Foster, V. Stavila, P. Haney, R.A. Kinney, V. Szalai, F. El. Gabaly, H.P. Yoon, F. Leonard, M.D. Allendorf, Science 2014, 343, 66 


\section{COMMUNICATION}

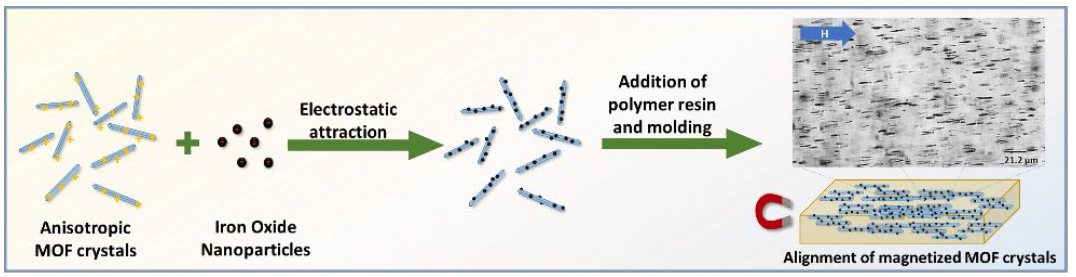

Fei Cheng, Ellis S. Marshall, Adam J. Young, Peter J. Robinson, JeanSebastien G. Bouillard, Ali M. Adawi, Nicolaas A. Vermeulen, Omar K. Farha, Michael R. Reithofer*, Jia Min Chin*

Page No. - Page No.

Magnetic Control of MOF Crystal Orientation and Alignment

Free-standing $\mathrm{NH}_{2}-\mathrm{MIL}-53(\mathrm{Al})$ and NU-1000 MOF crystals were magnetized by electrostatic adsorption of iron oxide nanoparticles onto their surfaces, and then magnetically aligned in different fluid matrices. MOF/polymer composites with aligned MOF crystals were prepared, and the importance of crystal orientation on MOF functionality was demonstrated using aligned NU-1000/Sylgard 184, which showed anisotropic fluorescence response to linearly polarized excitation. This general strategy for controlling MOF crystal orientation will open up new avenues for exploiting anisotropic MOF properties. 